\title{
HISTOPATHOLOGICAL AND IMMUNOHISTOCHEMICAL STUDIES IN EXPERIMENTAL HETEROPHYIASIS AND THE ROLE OF PRAZIQUANTEL AND AMINOGUANIDINE
}

\author{
By \\ SOAD M. M. NADA ${ }^{1}$, AFAF A. TAHA ${ }^{*}$, GHADA M. FATHY ${ }^{1}$, FATEN A. \\ MOHAMMED', MARWA O. ABDEL-AZIZ1 and HAYAM H. RASHED ${ }^{2}$ \\ Departments of Medical Parasitology ${ }^{1}$ and Pathology ${ }^{2}$, Faculty of Medicine, \\ Zagazig University, Egypt ( ${ }^{*}$ Correspondence: drafaf343@yahoo.com)
}

\begin{abstract}
Histopathological diagnosis was used to understand the pathological events associated with Heterophyes heterophyes (H. heterophyes) infection. CD3 and CD79 $\alpha$ antibodies had been used as markers for both $\mathrm{T}$ and $\mathrm{B}$ lymphocytes respectively. Immunohistochemical techniques had several advantages as remarkable sensitivity and specificity. This study aims to evaluate the roles of praziquantel (PZQ) and aminoguanidine (AG) treatment in $H$. heterophyes infected dogs pathologically and immunohistochemically. Study design included experimental infection of dogs with encysted metacercariae of $H$. heterophyes followed by treatment with PZQ and AG. Tissue samples were taken from small intestinal, liver, heart and lung of all groups for histopathological and immunohistochemical studies. Pathological changes were detected in infected tissues by histopathological examination. There was different degree of $\mathrm{CD} 79 \alpha+\mathrm{B}$ lymphocytic \& CD3+T lymphocytic infiltration detected in immuno-histochemical stained tissues. PZQ caused improvement of pathological changes in the small intestine. However the cellular inflammatory infiltration increased. There was reduction in inflammatory infiltration after intake of AG. Both PZQ and AG improved the pathological changes in the liver, heart and lung, while the cellular inflammatory infiltration increased after PZQ and reduced by AG. Moreover in the lung AG improves pulmonary congestion and alveolar wall thickness.
\end{abstract}

Keywords: Aminoguanidine, Histopathology, Heterophyes, Immunohistochemical, CD3, CD79 $\alpha$, praziquantel.

\section{Introduction}

Heterophyiasis is an intestinal illness caused by infection with $H$. heterophyes flukes. It's considered one of the fish-borne trematodes. Despite the considerable public health impact of food-borne trematodes, these diseases were among the most neglected of neglected tropical diseases (Torgerson, 2013). H. heterophyes is mainly prevalent in Egypt among the inhabitants of the northern part of the Delta particularly around the lakes Manzala and Borollus where favorable conditions exist for parasite propagation and transmission (Makhlouf et al, 1987). Heterophyes infection in man occurs from eating insufficiently cooked fish, the still-living metacercariae on reaching the small intestine emerge from their cysts, attach them to the mucosa and mature. They may cause superficial necrosis of the mucosa in these areas (Kang et al, 1983). Heterophyes infection diminished the host mucosal immunity to the flukes which may find their way easily across the intestinal wall to blood stream (Africa et al, 1937; Hamdy and Nicola, 1979). Ectopic infections do occur and most commonly involve the brain, spinal cord and heart (Africa et al, 1937, 1940; Chai et al, 1984; Zhang and Fan, 1990). Elsheikha (2007) reported that worms and eggs might cross the intestinal wall and spread to visceral organs such as the lymph nodes, liver and spleen. He suggested that under certain circumstances human, especially immunocompromised may be at risk of ectopic heterophyiasis.

Histo-pathological diagnosis was used to know pathological events associated with heterophyiasis (Ashour et al, 2014). It was often necessary to understand how the host responds to the pathogen as regard to the nature of the immune response; whether cellular or humoral and if cellular whether it's $\mathrm{T}$ helper 1 (Th1) or T helper 2 (Th2) polarized. Such determination is assessed by the application of immunohistochemical (IHC) 
studies (Gualco et al, 2010). Experimentally CD3 \& CD79 $\alpha$ antibodies were used as markers for both T \& B lymphocytes respectively (Sakamoto et al, 2013). Modern immuno-histochemical studies of paraffin tissue sections assessed the normal expression pattern of tissue antigens in cells and tissues, determine whether cellular antigens are lost and identify the cell types associated with an inflammatory lesion (Rehg et al, 2012). Immunohistochemical techniques have several advantages as remarkable sensitivity, specificity, applicability to routinely processed material (even if stored for long periods) and feasibility of an accurate correlation with the traditional morphological parameters (Chan, 2000).

Praziquantel (PZQ) is a broad spectrum anti-helminthic drug. It's the drug of choice for the food-borne trematodes, except for fascioliasis. It has an excellent safety profile (Jung et al, 1997). PZQ was used in treatment of human intestinal flukes. Taraschewski et al. (1986) evaluated the effect on Fasciolopsis buski and H. heterophyes, found that, PZQ at a dose of $15 \mathrm{mg} / \mathrm{kg}$, resulted in a very high cure rate of these infections. Aminoguanidine (AG) was described by Corbett et al. (1992) as a selective inhibitor of inducible nitric oxide synthase (iNOS), experimentally to evaluate effects upon parasitosis and inflammatory process to investigate the influence of the inhibition of nitric oxide (NO) production (Muro and Perez-Arellano, 2010).

The present study evaluated the roles of PZQ and AG treatment in $H$. heterophyes infection pathologically and immunohistochemically.

\section{Materials and Methods}

Type of study: Case control experimental study. The study was conducted from early June 2014 to late May 2015. Housing of dogs was carried out in Parasitology department, Faculty of medicine, Cairo University. Afterwards, the study was presumed in Parasitology and Pathology Departments, Faculty of Medicine, Zagazig University.
Animals and experimental design: The study included twenty male puppies of matched age (70 days old) and weight (about $4 \mathrm{~kg}$. each). Three stool samples were examined on consecutive days to ensure that they were free of other parasitic infections. Infected puppies were excluded.

The dogs were divided into 4 groups (5 dogs/each). The first (GI) was the control group; the second (GII) was infected with encysted metacercariae of $H$. heterophyes; the third group (GIII) was infected with encysted metacercariae of $H$. heterophyes and treated with PZQ after establishment of infection; the fourth group (GIV) was Infected and treated with AG. All animals were kept under strict hygiene conditions, housed in metallic cages, fed on milk and bread and kept under fixed appropriate conditions of housing and handling.

Drugs: 1-Praziquantel (PZQ): was obtained from EPICO Pharmaceutical Co., Egypt, in the form of "Distocide" tablets and given orally at a dose of $25 \mathrm{mg} / \mathrm{kg}$ for 5 days, and 2-Amino-guanidine (AG): was obtained from Sigma-Aldrich, St Louis, MO, USA. Aminoguanidine crystals were dissolved in $2 \mathrm{ml}$ of sterile normal saline and given orally at a dose of $25 \mathrm{mg} / \mathrm{kg}$ for 5 days.

Experimental infection: Infected Mugil cephalus and Tilapia species were collected from fish markets in Sharkia and Dakahlia Governorates. Then transferred to the laboratory and examined within few days (3-4) for viable $H$. heterophyes encysted metacercariae (EMC) in compressed snips from the fishes muscles (Elsheikha and Elshazly, 2008). The number of EMC was calculated per gram fish muscles to adjust about 250 EMC for each dog. Dogs were feed orally on infected fish muscles by means of forceps. Infection was determined by stool examination. Four weeks after treatment tissue samples were taken from small intestine, liver, lung and heart of all groups for histopathological and immunohistochemical studies. 
Measurements: Histopathological examination was performed on tissue specimens fixed in $10 \%$ formalin, washed, dehydrated in ascending grades of alcohol, cleared in xylene, embedded in paraffin wax, sectioned and stained with haematoxylin \& eosin (Liewellyn, 2009). Serial sections from the tissues were immunohistochemically stained for CD3+T \& CD79 $\alpha+B$ lymphocytes using the streptavidin-biotin peroxidase complex method (Adams, 1992). Immunohistochemical staining was performed using monoclonal rabbit anti-human CD3 antibody (Genemed, C 52-0003, South San Francisco, CA, USA) to detect CD3+ T lymphocytes, and monoclonal mouse anti-human CD79 $\alpha$ antibody (Genemed, C54-0003) to identify CD $79 \alpha+$ B lymphocytes. Briefly, slides were deparaffinised in xylene and hydrated through graded alcohols. Endogenous peroxidase activity was blocked using a $3 \%$ solution of hydrogen peroxidase in phosphate buffered saline (PBS) at room temperature for 10 minutes. After microwave treatment, primary antibody was applied for 30 minutes at room temperature and washed in PBS. Linking antibody and streptavidin-peroxidase complex were added consecutively for 10 minutes at room temperature and washed in PBS. The peroxidase activity was visualized with diaminobenzidine (Genemed, C100006) applied for $5 \mathrm{~min}$. Counter staining was done with Haematoxylin. Appropriate positive and negative controls were also labeled with the primary antibody and sections were examined by light microscopy.

Ethical considerations: The dogs were treated according to the research protocols following the recommendations of the $\mathrm{Na}$ tional Institutes of Health Guide for Care and Use of Laboratory Animals.

\section{Results}

Histopathological: In uninfected control dogs (GI), their intestinal sections revealed tall and slender villi, short and well-arranged crypts of Lieberkühn and normal features of submucosa and other layers (Fig. 1). In GII dogs infected with $H$. heterophyes there were villous atrophy and crypt hyperplasia (Fig. 2). Erosions were also observed (Fig. 3 ). In the lamina propria, there was marked inflammatory reaction especially, lymphocytic infiltration (Fig. 4). Goblet cells were markedly depleted (Fig. 5). In GIII dogs, after treatment with praziquantel, there was marked reduction in the pathological changes, restoration of normal villous architecture and goblet cells began to increase and return to normal (Fig. 6). However, the marked inflammatory cellular infiltration persisted (Fig. 7). In GIV dogs, after taking aminoguanidine, the inflammatory infiltration was markedly reduced (Fig. 8) and reduction in villous and crypt damage but there was erosion and marked goblet cell loss.

Immunohistochemical: CD79 $\alpha+\mathrm{B}$ lymphocytes were detected as an element of the inflammatory infiltrates, especially in the lamina propria of small intestinal villi of infected dogs (GII) which revealed marked degree of CD79 $\alpha+B$ lymphocytic infiltration (Fig. 9).While CD3+T lymphocyte infiltration was observed in a moderate degree in the same group (Fig. 10). In GIII dogs, there was marked degree of both CD3+T lymphocyte infiltration especially in sub-mucosal layer in (Fig. 11), and CD79 $\alpha+$ B lymphocytes especially in the small intestinal glands (Fig. 12). In GIV dogs, there was reduction in amount of both CD3+T lymphocytic infiltration with (-ve) CD79 $\alpha+B$ lymphocytic infiltrations (Figs. 13, 14).

Histopathological: Liver sections of infected dogs (GII) showed marked degree of intra-lobular degeneration and marked hepatocellular necrosis that was demonstrated as scattered foci of focal inflammation mainly lymphocytic, inside infected hepatocytes. Such findings were in contrast to those of (GIV) dogs which showed marked reduction in hepato-cellular necrosis and only showed mild degree of focal inflammation (Fig. 15). Other pathological changes in liver sections of (GII) dogs were marked ballooning degeneration of hepatocytes (Figs. 16). However, in (GIII), (GIV) dogs, the pathological 
features reduced and the architecture was restored nearly to normal and most of hepatocytes were more or less similar to those of control group.

Immunohistochemical: In liver sections of (GII) infected dogs there was mild CD79 $\alpha$ +B lymphocytic infiltrations, compared to moderate $\mathrm{CD} 3+\mathrm{T}$ lymphocyte infiltration. While in (GIII) dogs, both CD79 $\alpha+$ B lymphocytes and $\mathrm{CD} 3+\mathrm{T}$ lymphocytes increased to a marked degree (Fig. 17). This was in contrast to (GIV) dogs, in which both CD3+ T lymphocytes and CD79 $\alpha+$ B lymphocytes showed only low amounts inside hepatocytes.

Histopathological: Normal cardiac tissue with elongated cardiomyocytes and elongated nuclei was observed in GI. In cardiac sections of (GII) dogs, there were remarkable pathological changes like; severe myocardial degeneration, oedema and necrotic changes of cardiac muscles in (Figs. 18, 19). In (GIII) and (GIV) dogs, pathological changes like necrosis of cardiac fibers and myocardial degeneration were reversible and returned back to normal. However, inflammatory cellular infiltration persisted in (GIII). This was in contrast to (GIV) in which, inflammatory infiltration was markedly reduced. Besides, vascular changes like oedema and congestion were not improved in (GIII), while in (GIV), there was improvement of vasculature.

Immunohistochemical: In cardiac sections of (GIII) dogs, there was severe degree of both CD79 $\alpha+$ B lymphocytic and CD3 + T lymphocytic infiltrations as shown in (Figs. 20, 21). The cellular infiltration degree was much lowered in (GIV) after administration of AG in (Fig. 22). While in (GII), there was a moderate degree of $\mathrm{CD} 3+\mathrm{T}$ and $\mathrm{CD} 79 \alpha+$ B lymphocytic infiltration (Figs. 23).

Histopathological: In lung section of (GII) dogs, many pathological changes were observed like, marked thickness of the alveolar wall, vascular changes of pulmonary blood vessels, mainly congestion (Figs. 24, 25). Besides, the marked inflammatory cellular infiltration in (GIII) dogs, after treatment with praziquantel, some pathological abnormalities returned to normal like alveolar wall thickness as a result of death of the parasite. However, the inflammatory cellular infiltration persisted and congestion of pulmonary blood vessels was still of marked degree. In contrast to (GII \& GIII), in (GIV) there was resolution of many pathological changes; inflammatory cellular infiltration markedly reduced. Also, there was reduction of pulmonary congestion and alveolar wall thickness which returned nearly to normal.

Immunohistochemical: In lung sections of (GIII) dogs, there was increase especially in CD3+ T lymphocytic infiltration compared to (GII) dogs, as in (GII), there was a moderate degree of infiltration (Fig. 26). In (GIV) dogs, CD79 $\alpha+$ B lymphocytic infiltration was lowered to a mild degree compared to a moderate degree in (GII) infected dogs (Figs. 27, 28). In (GIV) no CD3+T lymphocytic infiltration was detected (Fig. 29).

\section{Discussion}

Heterophyiasis is a highly endemic disease in Egypt which may be asymptomatic or present with abdominal pain and diarrhea in infected humans (El-Kowrany et al, 1996). Their eggs, after being transferred by the blood stream, may produce eosinophilic granuloma in the heart, brain, and spinal cord in humans and animals. In Philippines, $15 \%$ of fatal heart disease might be a result of heterophyid myocarditis (Kim et al, 2016).

Pathological changes in heart, liver and kidney were suggested to be due to deposition of immune complexes in tissues. ElKowrany et al. (1996) found that the deposition of $H$. heterophyes antigen in blood vessel wall of liver and kidney detected by immuno-fluorescent test, supporting the idea of high level of antigenaemia. It was also attributed to chemically toxic substances from the parasite (Pica et al, 2003).

Histopathological, and immunohistochemical studies were performed in the present study to evaluate the roles of PZQ and AG 
in small intestine, liver, heart and lung tissues. In (GII) dogs infected with $H$. heterophyes, there were marked changes in the wall of their small intestines, the mucosal changes were characterized by villous atrophy \& crypt hyperplasia. This agreed with Hamdy and Nicola (1980), Shams-Eldin (2011). Similar pathological features have been reported in other heterophyid fluke infections, such as Metagonimus Yokogawai (Chai, 1979, Lee et al, 1981; Kang et al, 1983), Fibricola seoluensis (Lee et al, 1985) and Pygidiopsis summa (Seo el al., 1986). In (GIII), after intake of PZQ, marked improvement of pathological events was reported. The Same results were obtained by Chai et al. (2009) and Chai (2013). While in (GIV) AG caused reduction in villous and crypt damage, inflammatory alterations. Kołodziej-Sob-ocińska et al. (2006) reported that mice experimentally infected with Trichinella spiralis showed inhibition of NO production by $A G$ prevents the intestinal mucosa damage due to cytotoxicity caused by NO inhibited in mice intestines Itreated with AG. Khan et al. (1997) proved the protective role of AG against small bowel necrosis in mice experimentally infected with Toxoplasma gondii during the acute stage of infection. Dikopoulos et al. (2001) reported that inhibition of nitric oxide synthesis by AG increases intestinal damage in experimental colitis and that NOS inhibition by AG is not beneficial in acute intestinal inflammation.

Erosions were observed in the infected group (GII) and also in (GIV). Similar results were reported by Tiso \& Schechter (2015) who demonstrated that nitric oxide (NO) plays an important role in maintaining the integrity of the small intestine. In (GIII), ulceration was not a reported as side effect of PZQ. Depletion of goblet cells was detected in (GII). Ashour et al. (2014) also reported marked goblet cell loss in $H$. heteropyes infection. Moreover, the current study demonstrated marked goblet cell loss in (GIV). This was in accordance with Val- lance et al. (2004) who indicated that NO plays an important role in maintaining goblet cell numbers and function. On the other hand, in (GIII) after intake of PZQ, goblet cells were restored to normal level. This agreed with Lee et al. (1989a) and ShamsEldin (2011).

Marked inflammatory reaction (especially lymphocytic) was detected in lamina propria of (GII) infected dogs, which agreed with Shams-Eldin (2011) and Ashour et al. (2014). In (GIV) after AG, there was reduction of inflammatory infiltration that agreed with Griffiths et al. (1993) and Zhang et al. (2001).

In the intestinal sections of (GII), sever degree of CD79 $\alpha+B$ lymphocytes were detected, while CD3+ T lymphocyte infiltration was observed in a moderate degree. In (GIII) post-PZQ treatment showed marked increase in CD3+T, and CD79 $\alpha+\mathrm{B}$ lymphocyte infiltration. Death of the parasite by the action of PZQ results in exposure to internal parasite antigens and so host humoral and cellular immune responses can be active following the exposure of these antigens (Zhang and Mutapi, 2006). In (GIV) after intake of $A G$, there was reduction in the amount of both CD3+ T lymphocytic infiltration with absent of CD79 $\alpha+B$ lymphocytic infiltrations. Anti-inflammatory effect of AG demonstrated in the current study was confirmed by Beytut et al. (2011) who reported the reduction in the amount of inflammatory infiltration for both CD3 and CD79 $\alpha$ markers after administration of AG in Fasciola hepatica experimentally infected rats. Farhad et al. (2011) in cats evaluated anti-inflammatory effect of AG as a selective inducible nitric oxide synthase inhibitor and concluded that, AG reduced the periapical inflammation degree in teeth. The current study showed different distribution between CD3 \& CD79 $\alpha$ markers in intestinal sections of (GII) dogs. Cerutti (2008) reported the predominance of $\mathrm{B}$ lymphocytes in the lamina propria of the small intestine. $\mathrm{Li}$ et al. (2012) reported that $\mathrm{T}$ cells were found 
scattered in the layers of the small intestine, within intestinal epithelium or at the periphery of Peyer's patches.

In the current study, liver sections of dogs (GII) showed marked intra-lobular degeneration degree and hepato-cellular necrosis which appeared as scattered foci of focal inflammation inside infected hepatocytes. Other reported pathological changes were marked ballooning degeneration of hepatocytes, bands of fibrosis and marked disturbance of normal liver architecture. This agreed with Shalaby et al. (2003) who reported liver congestion with focal necrosis in extra-intestinal heterophyiasis. Elsheikha (2007) reported that extra-intestinal heterophyiasis caused hydropic degeneration of hepatocytes, and extramedullary haematopoiesis in spleen. The present study showed that in (GIII), after PZQ treatment, the pathological features reduced. Those results were in accordance with Barros et al. (2009) and El-Lakkany et al. (2012) who proved reversal of hepatic fibrosis after PZQ therapy in experimental schistosomiasis.

In (GIV), marked reduction of hepatocellular necrosis was demonstrated and there was only mild degree of focal inflammation. Gargili et al. (2004) reported that AG reduced microscopic damage and injury of multiple organs. They also confirmed that (iNOS) inhibition can protect the tissue from damage in mice infected with of Toxocara canis larvae. De-mirci et al. (2006) observed significant differences between $T$. canis infected and AG treated animals, and concluded that, AG decreased pathological changes in the liver and lung tissues. Khan et al. (1997) concluded that during acute $T$. gondii infection, AG prevented hepatic degeneration of the acutely infected mice. Ahmed et al. (2011) evaluated the possible hepato-protective effect of AG in comparison with silymarin. They concluded that, AG treated rats revealed marked reduction in the hepatic lesions. Liu et al. (2002) found that iNOS inhibition resulted in a marked reduction in liver injury and nitric oxide played an active role in progression of liver fibrosis and hepato-celluar damage, and that serum levels of nitric oxide were significantly higher in liver cirrhosis patients. But, Hesse et al. (2000) found that NO was necessary to limit hepatocyte damage and to block nitric oxide production with its profound harmful effect.

In the current study, liver sections of (GII) showed mild CD79 $\alpha+B$ lymphocytic infiltrations compared to moderate $\mathrm{CD} 3+\mathrm{T}$ lymphocyte infiltration. In (GIII) dogs, CD79 $\alpha+B$ lymphocytes \& CD3+T lymphocytes markedly increased. Also, Mduluza et al. (2009) showed increase in cellular activity after PZQ therapy due to exposure of released antigens to host im-mune system after worm destruction by PZQ. In (GIV), CD3+T lymphocytes \& CD79 $\alpha+\mathrm{B}$ lymphocytes were low inside hepatocytes. Anti-inflammatory effect of AG in the current study agreed with Korhonen et al. (2005) who found role of nitric oxide in mediating inflammatory response and nitric oxide synthase inhibitors in decreasing cellular infiltration.

In the present study, in cardiac sections of (GII) there were remarkable pathological changes like; severe myocardial degeneration, oedema and necrotic changes of cardiac muscles. Such findings were in agreement with Shams-Eldin (2011). In (GIII) after treatment with PZQ, pathol- ogical changes returned back to normal. However, inflammatory cellular infiltration persisted and this agreed with Singhi (2011) who found that using PZQ for neurocysticercosis induced intense reactive inflammation as a result of death of cysticerci. On the other hand, in (GIV) there was marked reduction in the degree of inflammatory infiltration. The effect of AG in reducing inflammatory infiltration agreed with Mouzaoui et al. (2012) who proved the effect of AG in attenuation of neutrophilic influx.

Vascular changes like congestion and oedema were high in both (GII) and (GIII). Improvement of vascular cardiac changes 
was observed in (GIV) after AG administration. This agreed with Chan et al. (2006) who showed that AG improved cardiovascular function in experimental hypertensive rats. Liu et al. (2006) found that nitric oxide negatively regulated cardiac contractile function and was involved in some type of cardiac dysfunctions as ischemic heart failure and found that inhibition of (iNOS) restored the heart contractile function. But, Gomez et al. (2015) reported that AG had no effect on vascular tone in myocardial infarction. In the cardiac sections (GII), there was a moderate degree of CD3+T \& CD79 $\alpha+$ B lymphocytic infiltrations while in (GIII) with marked degree of $\mathrm{CD} 79 \alpha+\mathrm{B}$ lymphocytic \& CD3+T lymphocytic infiltra-tions. The increase in cellular inflamma-tory infiltration after PZQ therapy was reported by Silveira-Lemos et al. (2013) in patients with S. mansoni. Inflammatory cellular infiltration degree of was ma-rkedly lowered in (GIV). Cigremis et al. (2006) reported the beneficial role of AG in reducing inflammatory cellular infiltration and reversing myocardial fibrosis.

In this study, lung sections of (GII) dogs showed many pathological changes like thickening of alveolar wall, congestion of pulmonary blood vessels and cellular inflammatory infiltration. Changes were attributed to dense fibrosis and inf-lammatory process associated with infection (Beigelman-Aubry et al, 2012). Pat-hological lung changes due to parasitosis were reported (Lahiri, 1993; Kuzucu, 2006; Chitkara and Krishna, 2006; Vijayan, 2008). In (GIII) after treatment with PZQ, some pathological abnormalities improved. Lee et al. (1989 b) evaluated the effect of PZQ treatment on pulmonary lesions of Paragonimus iloktsuenensis rats infected found that PZQ was highly effective not only by killing wor-ms but also excellent resolution capacity of pulmonary tissues. But, in this group, congestion of pulmonary blood vessels was still of marked degree. Also, Flisser and McLaren (1989) reported that haemorrhages in lung tissues of $S$. mansoni infection mice treated with PZQ. This occurred due to parasite death exposure to parasite antigen and release of its derived enzymes. Resolution of pathologcal changes were observed in (GIV). Hu et al. (1999) found the role of AG as a nitric oxide synthase inhibitor, in resolution of bleomycin induced-lung injury in rats. They showed that AG ameliorated alveolitis, resolved pulmonary fibrosis caused by nitric oxide and reduced inflammatory response. Muro and Perez-Arellano (2010) reported that of NO inhibition by iNOS in toxocariosis experimental model decreased harmful effects upon host mainly lung vascular alterations.

In lung sections (GIII) there was incre-ase in $\mathrm{CD} 3+\mathrm{T}$ lymphocytic infiltration, which agreed with Brown et al. (2005) who reported that PZQ therapy induced damage in the worm tegument which resulted in exposure to the parasite antigens that induce strong type 2 immune response in Schistosoma mansoni infected Ugandan adults. In (GIV), there was a mild degree of CD79 $\alpha+B$ lymphocytic infiltration, compared to a moderate degree in (GII). Also, CD3+T lymphocytic infiltration was markedly reduced. The effect of AG in reducing inflammatory infiltration reported in the present study agreed with Worrall et al. (1995).

\section{Conclusion}

PZQ is more effective in treatment of the intestinal lesions as it improved the pathological lesions. AG is more effective in treatment of ectopic lesions of $H$. heterophyes in dogs as it has an anti-inflammatory role.

\section{References}

Ahmed, AF, Mahmoud, MF, Ouf, MA, ElFathaah, EA, 2011: Aminoguanidine potentiates the hepatoprotective effect of silymarin in CCL4 treated rats. Ann. Hepatol. 2:207-15.

Adams, JC, 1992: Biotin amplification of biotin and horseradish peroxidase signals in histochemical stains. J. Histochem. Cytochem. 40:1457-63. Africa, CM, Garcia, EY, De Leon, W, 1937: Intestinal heterophyiasis with cardiac involvement: a contribution to the etiology of heart failure. Philipp. J. Pub. Hlth. 1:1-35.

Africa, CM, De Leon, W, Garcia, EY, 1940: 
Visceral complications in intestinal heterophyidiasis of man. Acta. Med. Philipp. Monogr. Ser. 1:1-325.

Ashour, DS, Othman, AA, Radi, DA, 2014: Insights into regulatory molecules of intestinal epithelial cell turnover during experimental infection by Heterophyes heterophyes. Exp. Parasitol., 143:48-54.

Barros, LA, Costa-Silva, M, Biolchini, CL, Neves, RH, Machado-Silva, JR, 2009: Effect of praziquantel administration on hepatic stereology of mice infected with Schistosoma mansoni and fed a low-protein diet. Braz. J. Med. Biol. Res. 9:812-5.

Beigelman-Aubry, C, Godet, C, Caumes, E, 2012: Lung infections: the radiologist's perspective. Diagn. Interv. Imag. 6:431-40.

Beytut, E, Akca, A, Halil İbrahim Gokce, HI, 2011: Pathological and immunohistochemical evaluation of the effects of interferon gamma IFN- $\gamma$ and aminoguanidine in rats experimentally infected with Fasciola hepatica. Turk. J. Vet. Anim. Sci. 4: 243-253.

Brown, M, Mawa, PA, Joseph, S, Bukusuba, J, Watera, C, et al, 2005: Treatment of Schistosoma mansoni infection increases helminthspecific type 2 cytokine responses and HIV-1 loads in co-infected Ugandan adults. J. Infect. Dis. 10:1648-57.

Cerutti, A, 2008: Location, location, location: B-cell differentiation in the gut lamina propria. Mucosal Immunol. 1:8-10.

Chai, JY, 1979: Study on Metagonimus yokogawai Katsurada, 1912 in Korea: V. Intestinal pathology in experimentally infected albino rats. Seol. J. Med. 2:104-17.

Chai, JY, 2013: Praziquantel Treatment in Trematode and Cestode Infections: An Update. Infect. Chemother. 1:32-43

Chai, JY, Seo, BS, Lee, SH, 1984: Studies on intestinal trematodes in Korea XI. Two cases of human infection by Heterophyes heterophyes nocens. Kisaengchunghak Cha-pchi. 22:37-42.

Chai, JY, Shin, EH, Lee, SH, Rim, HJ, 2009: Foodborne intestinal flukes in Southeast Asia. K. J. Parasitol. 47:69-102.

Chan JK, 2000: Advances in immunohistochemistry: impact on surgical pathology practice. Semin. Diagn. Pathol. 17:170-7.

Chan, V, Hoey, A, Brown, L, 2006: Impro-ved cardiovascular function with aminoguanidine in
DOCA-salt hypertensive rats. Br. J. Pharmacol. 7:902-8.

Chitkara, RK, Krishna, G, 2006: Parasitic pulmonary eosinophilia. Semin. Respir .Crit. Care Med. 2:171-84.

Cigremis, Y, Parlakpinar, H, Polat, A, Colak, C, Ozturk, F, et al, 2006: Beneficial role of aminoguanidine on acute cardiomyopathy related to doxorubicin-treatment. Mol. Cell Biochem. 1/2:149-54.

Corbett, JA, Tilton, RG, Chang, K, Hasan, KS, Ido, Y, et al, 1992: Aminoguanidine, a novel inhibitor of nitric oxide formation, prevents diabetic vascular dysfunction. Diabetes 41:552-6.

Demirci, C, Gargili, A, Kandil, A, Ceti-nkaya, H, Uyaner, I, et al, 2006: Inhibition of inducible nitric oxide synthase in murine visceral larva migrans: effects on lung and liver damage. Chin. J. Physiol. 6:326-34.

Dikopoulos, N, Nüssler, AK, Liptay, S, Bachem, M, Reinshagen, M, et al, 2001: Inhibition of nitric oxide synthesis by aminoguanidine increases intestinal damage in the acute phase of rat TNB-colitis. Eur. J. Clin .Invest. 3: 234-9.

El-Kowrany, SE, AbdEl-Ghaffar, AE, Ghoraba, HM, 1996: Experimental heterophyiasis: Effect of immuno-supression on the course of the disease. Egypt. J. Med. Sci. 2: 481-97.

El-Lakkany, NM, Hammam, OA, El-Maadawy, WH, Badawy, AA, Ain-Shoka, A, et al, 2012: Anti-inflammatory/anti-fibrotic eff-ects of the hepatoprotective silymarin and the schistosomicide praziquantel against Schistosoma mansoni-induced liver fibrosis. Parasit. Vectors 5:114.

Elsheikha, HM, Elshazly, AM, 2008: Preliminary observations on infection of brackish and fresh water fish by heterophyid encysted metacercariae in Egypt. Parasitol. Res. 103:971-977.

Elsheikha, HM, 2007: Heterophyosis: risk of ectopic infection. Vet. Parasitol., 3-4:341-2.

Farhad, AR, Razavi, S, Jahadi, S, Saatchi, M, 2011: Use of aminoguanidine, a selective inducible nitric oxide synthase inhibitor, to evaluate the role of nitric oxide in periapical inflammation. J. Oral Sci. 2:225-30.

Flisser, A, McLaren, DJ, 1989: Effect of praziquantel treatment on lung-stage larvae of $S \mathrm{ch}$ istosoma mansoni in vivo. Parasitology 2:20311. 
Gargili, A, Demirci, C, Kandil, A, Cetin-kaya, H, Atukeren, P et al, 2004: In vivo inhibition of inducible nitric oxide and evaluation of the brain tissue damage induced by Toxocara canis larvae in experimentally infected mice. Chin. J. Physiol. 4:189-96.

Gomez, JF, Cardona, K, Trenor, B, 2015: Lessons learned from multi-scale modeling of the failing heart. J. Mol. Cell. Cardiol. 89:14659.

Griffiths, MJ, Messent, M, MacAllister, R J, Evans, TW, 1993: Aminoguanidine selectively inhibits inducible nitric oxide synthase. Br. J .Pharmacol. 3:963-8.

Gualco, G, Weiss, LM, Bacchi, CE, 2010: A review. Appl. Immunohistochem. Mol. Morphol. 18:301-10.

Hamdy, EJ, Nicola, E, 1979: Studies on Heterophyiasis on the histopathology of the small intestine in experimentally infected animals. J. Egypt. Med. Assoc. 14, 224-229.

Hamdy, EI, Nicola, E, 1980: On the histopathology of the small intestine in animals experimentally infected with $H$. heterophyes. J. Egypt. Med. Assoc. 63:179-84.

Hesse, M, Cheever, AW, Jankovic, D, Wynn, TA, 2000: NOS-2 mediates the protective antiinflammatory and antifibrotic effects of the Th1inducing adjuvant, IL-12, in a Th2 model of granulomatous disease. Am. J. Pathol. 3: 945-55. Hu, J, Xu, Q, Li, B 1999: The effect of aminoguanidine, a nitric oxide synthase inhibitor, on bleomycin-induced lung injury in rats. Zhonghua Jie He He Hu Xi Za Zhi. 1:51-3.

Jung, H, Medina, R, Castro, N, Corona, T, Sotelo, J, 1997: Pharmacokinetic study of praziquantel administered alone and in combination with cimetidine in a single-day therapeutic regimen. Antimicrob. Agents Chem- other. 6:12569.

Kang, SY, Cho, SY, Chai, JY, Lee, JB, Jang, DH, 1983: A study on intestinal lesions of experimentally re-infected dogs with Metagonimus yokogawai. Kisaengchunghak Chapchi. 1:58-73. Khan, IA, Schwartzman, JD, Matsuura, T, 1997: Dichotomous role for nitric oxide during acute Toxoplasma gondii infection in mice. Proceed. Nat. Acad. Sci. USA. 25: 13955-60.

Kim, K, Weiss, LM Tanowitz, HB, 2016: Murray and Nadel's Textbook of Respiratory Medicine. $6^{\text {th }}$ ed., by John F. Kennedy Blvd. Ste. 1800. Philadelphia.
Kołodziej-Sobocińska, M, Dziemian, E, Machnicka-Rowińska, B, 2006: Inhibition of nitric oxide production by aminoguanidine influences number of Trichinella spiralis parasites in infected low responders C57BL/6 and high responders BALB/c mice. Parasitol. Res. 2:194-6.

Korhonen, R, Lahti, A, Kankaanranta, H, Moilanen, E, 2005: Nitric oxide production and signaling in inflammation. Curr. Drug Targets Inflamm. Allergy, 4:471-9.

Kuzucu, A, 2006: Parasitic diseases of the respiratory tract. Curr. Opin. Pulm. Med. 3: 212-21. Lahiri, K, 1993: Parasitic infections of the respiratory tract diagnosis and management. J. Postgrad. Med. 3:144-8.

Lee, JB, Chi, JG, Lee, SK, Cho, SY, 1981: Study on the pathology of metagonimiasis in experimentally infected cat intestine. Korean, J. Parasit. 2:109-29.

Lee, SH, Kim, SY, Han, YC, Lee, YS, Hong, S T, et al, 1989b: Effect of praziquantel treatment on pulmonary lesions of rats infected with Paragonimus iloktsuenensis. Korean, J. Parasit. 2: 119-30.

Lee, SH, Kim, BI, Hong, ST, Sohn, WM, Chai, JY, 1989a: Observation of mucosal pathology after praziquantel treatment in experimental Fibricola seoulensis infection in rats. Korean J. Parasitol. 1:35-40.

Lee, SH, Yoo, BH, Hong, ST, Chai, JY, Seo, B S, et al, 1985: A histo-pathological study on the intestine of mice and rats experimentally infected with Fibricola Seoulensis. Korean J. Parasit.1:58-72.

Li, Z, Zhang, C, Zhou, Z, Zhang, J, Zhang, J, Tian, Z, 2012: Small intestinal intra-epithelial lymphocytes expressing CD8 and T cell receptor $\gamma \delta$ are involved in bacterial clearance during Salmonella enterica serovar Typhimurium infection. Infect. Immun. 2: 565-74.

Liewellyn, BD, 2009: Nuclear staining with alum-hematoxylin. Biotech. Histochem. 84:15977.

Liu, H, Gaskari, SA, Lee, SS, 2006: Cardiac and vascular changes in cirrhosis: pathogenic mechanisms. World J. Gastroenterol. 6: 837-42.

Liu, TH, Robinson, EK, Helmer, KS, West, S D, Castaneda, AA, et al, 2002: Does up regulation of inducible nitric oxide synthase play a role in hepatic injury? Shock 6:549-54.

Makhlouf, LM, Abou Zakham, A, el-Kholy, 
EI, Abou-Shady, AF, Abdel-Magied, S, 1987: Heterophyids of some fresh water fish from Mansoura. Egypt. J. Egypt. Soc. Parasitol., 17: 573-576.

Mduluza, T, Mutapi, F, Ruwona, T, Kaluka, D, Midzi, N, et al, 2009: Similar cellular responses after treatment with either praziquantel or oxamniquine in Schistosoma mansoni infection. Malawi Med. J. 4:176-82.

Mouzaoui, S, Rahim, I, Djerdjouri, B, 2012: Aminoguanidine and curcumin attenuated tumor necrosis factor TNF- $\alpha$-induced oxidative stress, colitis and hepatotoxicity in mice. Int. Immunopharmacol. 1:302-11.

Muro, A, Pérez-Arellano, JL, 2010: Nitric oxide and respiratory helminthic diseases. J. Biomed. Biotechnol. 2010:1-8.

Pica R, Castellano G, Cilia C, Errico FP, 2003: Intestinal fluke infections: The heterophyids. Clin Ter. 154, 1:61-3.

Rehg, JE, Bush, D, Ward, JM, 2012: The utility of immuno-histochemistry for the identification of hematopoietic and lymphoid cells in normal tissues and interpretation of proliferative and inflammatory lesions of mice and rats. Toxicol. Pathol. 2:345-74.

Sakamoto, KP, de Melo, GD, Machado, GF, 2013: $T$ and $B$ lymphocytes in the brains of dogs with concomitant seropositivity to three pathogenic protozoans: Leishmania chagasi, Toxoplasma gondii and Neospora caninum. B.M.C. Res. Notes 6:1-8.

Seo, BS, Cheong, SK, Chai, JY, Lee, SH, Lee, JB, 1986: Histopathology of Small Intestines of Rats and Mice Experimentally Infected with Pygidiopsis summa. Seoul J. Med. 2:12534.

Shalaby, SI, Hassan, NR, El-Mahdy, MM, 2003: Parasitological and pathological studies on extra-intestinal heterophyiasis. J. Parasitic Dis.1:39-46.

Shams-Eldin, S, 2011: Histopathological, histochemical, and immunohistochemical studies of experimental heterophyiasis in dogs and the protecting role of praziquantel. P.U.J. 2:185-92.
Silveira-Lemos, D, Fernandes Costa-Silva, M, Cardoso de Oliveira, A, Azevedo Batista, M, et al, 2013: Cytokine Pattern of T Lymphocytes in Acute Schistosomiasis mansoni patients following treated praziquantel therapy. J. Parasitol. Res., 2013:1-13.

Singhi, P, 2011: Neurocysticercosis. Ther. Adv. Neurol. Disord., 2: 67-81.

Taraschewski, H, Mehlhorn, H, Bunnag, D, Andrews, P, Thomas, H, 1986: Effects of praziquantel on human intestinal flukes Fasciolopsis buski and Heterophyes heterophyes. Zentralbl. Bakteriol. Mikrobiol. Hyg. 4:54250.

Tiso, M, Schechter, AN 2015: Correction: Nitrate Reduction to Nitrite, Nitric Oxide and Ammonia by Gut Bacteria under Physiological Conditions. PLoS One, 5:1-18.

Torgerson, PR, 2013: One world health: socioeconomic burden and parasitic disease control priorities. Vet. Parasitol. 3, 4:223-32.

Vallance BA, Dijkstra G, Qiu B, van der Waaij L, van Goor H, et al, 2004: Relative contributions of NOS isoforms during experimentalcolitis: endothelial-derived NOS maintains mucosal integrity. Am. J. Physiol.

Gastrointes. Liver Physiol. 287, 4:G865-74.

Vijayan, V.K. 2008: Tropical parasitic lung diseases. Indian J. Chest Dis. Allied Sci. 1: 4966.

Worrall, NK, Lazenby, WD, Misko, TP, Lin, TS, Rodi, CP, et al, 1995: Modulation of in vivo reactivity by inhibition of inducible nitric oxide synthase. J .Exp. Med., 1:63-70.

Zhang, GL, Wang, YH, Teng, HL, Lin, Z, 2001: Effects of aminoguanidine on nitric oxide production induced by inflammatory cytokines and endotoxin in cultured rat hepatocytes. World J. Gastroenterol., 3:331-4.

Zhang, P, Mutapi, F, 2006: IgE: a Key Antibody in Schistosoma Infection. Electronic J. Biol. 1:11-4.

Zhang, SL, Fan, GH, 1990. Case report brain abscess caused by heterophyid eggs. Chin. J. Parasitol. Parasit. Dis. 8:178-83.

\section{Explanation of figures}

Fig. 1: T. S. of small intestine in (GI) dogs, stained with Haematoxylin and Eosin( H\&E), showing normal villous architecture, X 100

Fig. 2: T. S. of small intestine of (GII) dogs, stained with Haematoxylin and Eosin, showing villous atrophy and crypt hyperplasia, $\mathrm{X} 100$

Fig. 3: T. S. in small intestine of (GII) dogs, stained with (H\&E) showing marked erosions of intestinal villi, X 100

Fig. 4: T. S. of small intestine of (GII) dogs, stained with (H\&E), showing marked lymphocytic infiltration inside intestinal villi , $\mathrm{X} 400$ 
Fig. 5: T. S. in small intestine section of (GII) dogs showing marked loss of goblet cells and marked cellular inflammatory infiltration, $\mathrm{X} 400$

Fig. 6: T. S. in small intestine section of (GIII) dogs, , stained with (H\&E), showing restoration of normal villous architecture

Fig. 7: Persistence of inflammatory infiltration in (GIII) dogs, stained with (H\&E), X 400

Fig. 8: Marked reduction in inflammatory infiltration in small intestinal section of (GIV) dogs, stained with (H\&E), X400

Fig. 9: T. S. in small intestinal villi of (GII) dogs showing marked CD79 $\alpha+$ B lymphocytic infiltration, X400

Fig. 10: Transverse section in small intestinal villi of (GII) dogs showing moderate CD3+ T lymphocytic infiltration, X400

Fig. 11: T. S. of small intestinal crypts showing marked CD3+ T lymphocytic infiltration in the submucosa of (GIII) dogs, X400

Fig. 12: T. S. of small intestinal glands showing marked CD79+ $\alpha$ B lymphocytic infiltration in (GIII) dogs, X400

Fig. 13: Mild CD3+ T lymphocytic infiltration in small intestinal sections of (GIV) dogs, X400

Fig. 14: T. S. in intestinal glands showing negative CD79+ $\alpha$ B lymphocytic infiltration in small intestinal sections of (GIV) dogs, X400

Fig. 15: Liver section in (GIV), stained with H\&E, showing focus of hepatocelluar necrosis, $\mathrm{X} 400$

Fig. 16: Liver section of (GII) dogs stained with H\&E, showing marked ballooning degeneration of hepatocytes , X400

Fig. 17: Marked CD79+ $\alpha$ B lymphocytic infiltration inside hepatocytes and around portal tract in liver sections of(GIII) dogs, X400

Fig. 18: Longitudinal section of heart of (GII) dogs stained with (H\&E), showing severe myocardial degeneration, X100

Fig. 19: Longitudinal section of heart of (GII) dogs showing marked necrotic changes, as well as oedema, X100

Fig. 20: Marked CD79 $\alpha+$ B lymphocytic infiltration around cardiac blood vessel which is located in-between muscle fibers of (GIII) dogs, X400

Fig. 21: Marked CD3+ T lymphocytic infiltration around cardiac blood vessel which is located in-between muscle fibers of (GIII) dogs, $\mathrm{X} 400$

Fig. 22: Mild CD3+ T lymphocytic infiltration around cardiac blood vessel which is located in-between muscle fibers of (GIV) dogs, $\mathrm{X} 400$

Fig. 23: Moderate CD79 $\alpha+$ B lymphocytic infiltration inside cardiac muscle fibers of (GII) dogs, X100

Fig. 24: Lung section of (GII) dogs stained with H\&E, showing marked thickening of alveolar wall , X100

Fig. 25: Lung section of (GII) group stained with H\&E, showing marked congestion of pulmonary blood vessels, X400

Fig. 26: Moderate CD3+ T lymphocytic infiltration inside alveolar wall of lung sections of (GII) dogs, X400

Fig. 27: Mild CD79+ $\alpha$ B lymphocytic infiltration around pulmonary blood vessel in lung sections of (GIV) dogs, X400

Fig. 28: Moderate CD79+ $\alpha$ B lymphocytic infiltration inside alveolar wall in lung sections of (GII) dogs, X400

Fig. 29: Absent (-ve) CD3+ T lymphocytic infiltration in lung sections of (GIV) dogs, X400.
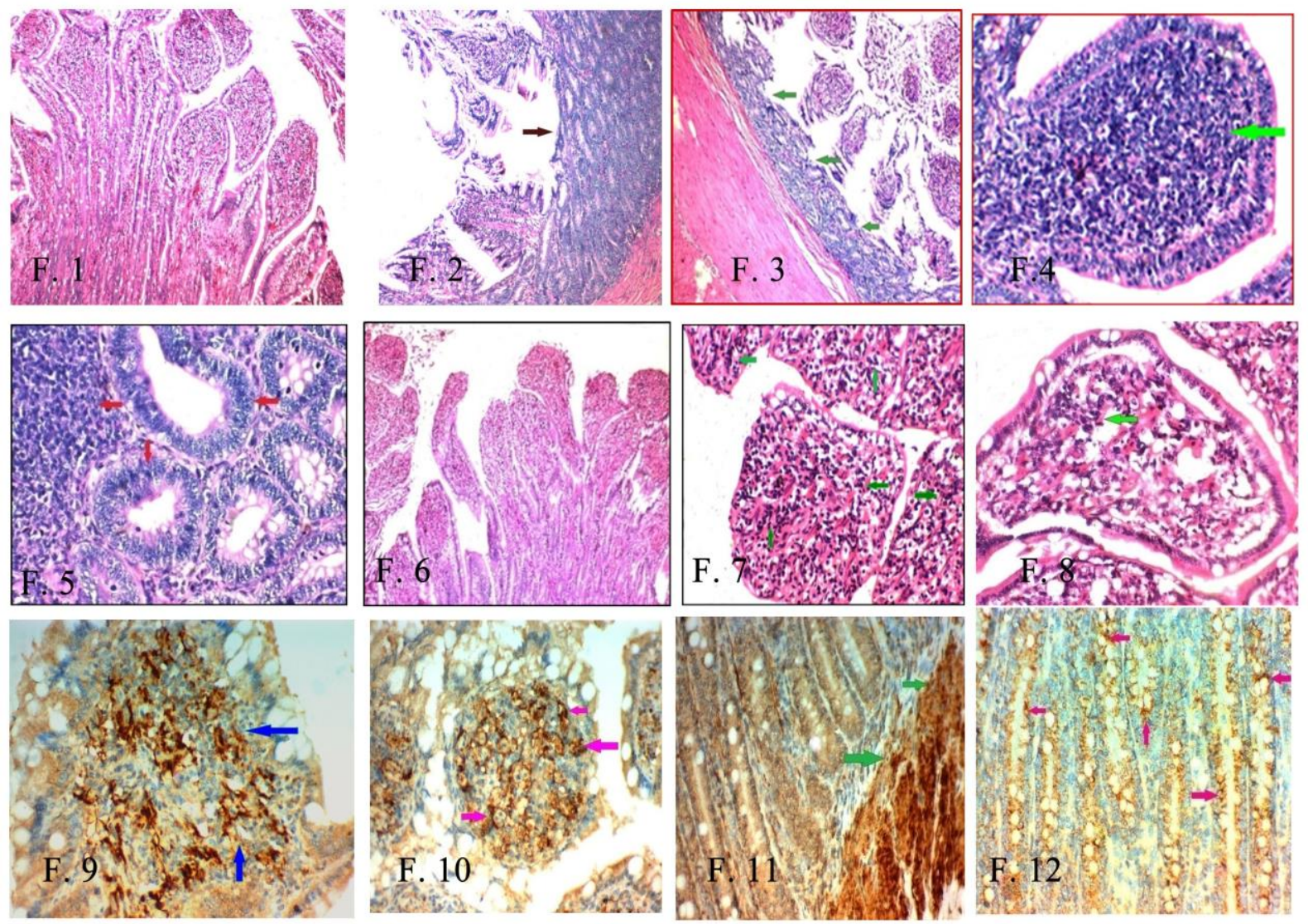

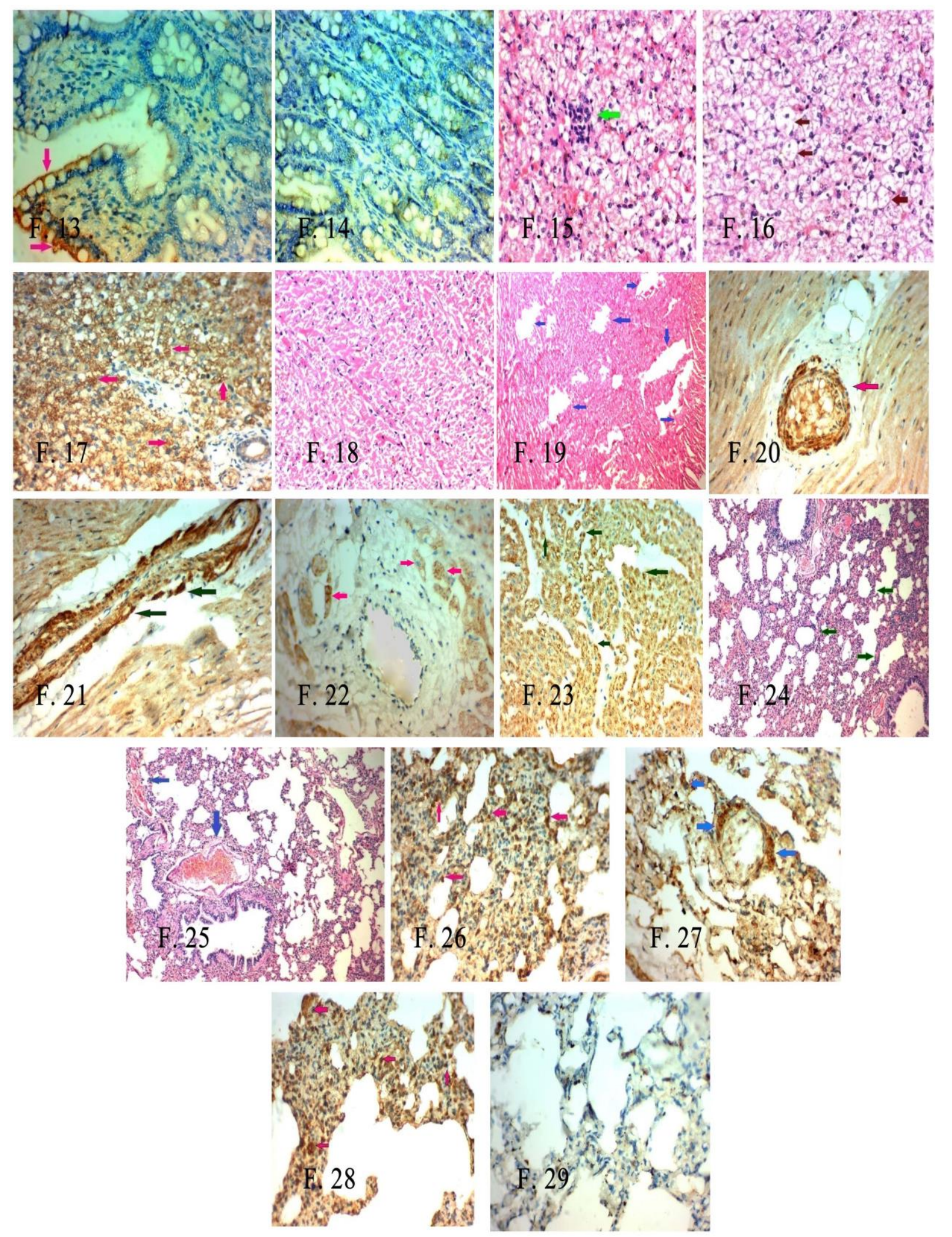